\title{
Short communication: Application of proteomics for characterization of caseinomacropeptide isoforms before and after desialidation
}

\author{
Anne Vuholm Sunds, ${ }^{*}$ Nina Aagaard Poulsen, and Lotte Bach Larsen \\ Department of Food Science, Aarhus University, Blichers Allé 20, PO Box 50, DK-8830 Tjele, Denmark
}

\section{ABSTRACT}

Caseinomacropeptide (CMP) is an important polypeptide found in cheese whey that has various biological activities and functional properties. Because sialylations play an important role in the functional properties of CMP, the aim of the present work was to characterize CMP isoform heterogeneity in a commercial glycosylated CMP (gCMP) isolate using liquid chromatography- and gel-based proteomics before and after desialidation. Using 2-dimensional gel electrophoresis (2-DGE), we observed a shift in isoelectric point (pI) after enzymatic desialidation, indicating that sialylated gCMP were located at pI 3.0 to 3.1 , but desialylated gCMP had repositioned to pI 3.7 to 3.9. We used liquid chromatography/mass spectroscopy (LC-ESI/MS) for further analysis of the glycan chains of gCMP. In total, we identified $19 \mathrm{CMP}$ isoforms, of which 13 were glycosylated and 6 were nonglycosylated. We also detected 3 genetic variants (A, B, and E), with up to 3 glycosylations attached per gCMP. Further, we identified up to 4 isomers, reflecting different sites of glycosylation in the peptide backbone of these isoforms. The dominating gCMP isoform of genetic variant $\mathrm{E}$ appeared to contain $4 \mathrm{~N}$-acetyl-neuraminic acid (NeuAc) residues, whereas the dominating gCMP isoforms of variants $\mathrm{A}$ and B appeared to contain 2 NeuAc. The identification revealed conversions of isoforms containing different combinations of genetic variants and degrees of glycosylation, sialylation, and phosphorylation to various desialylated counter-isoforms. The content of sialylated gCMP relative to the total CMP content was 37\% (wt/ wt), which decreased to $1.5 \%$ after sialidase treatment, indicating $96 \%$ effectivity of the desialidation. This approach can be valuable for future functionality studies specifically addressing the importance of NeuAc.

Key words: caseinomacropeptide, posttranslational modification, desialidation, reverse-phase LC-ESI/MS

Received March 13, 2019.

Accepted May 23, 2019.

*Corresponding author: annesunds@food.au.dk

\section{Short Communication}

The hydrophilic 64-amino acid polypeptide known as caseinomacropeptide (CMP) or glycomacropeptide is the C-terminal peptide released from bovine $\kappa$-casein during rennet-induced cheese production. Chymosin cleaves $\kappa$-casein at $\mathrm{Phe}^{105}-\mathrm{Met}^{106}$, resulting in the hydrolytic products para-k-casein (residues 1-105) and CMP (residues 106-169). Preparation of CMP isolate from cheese whey is of growing interest due to its reported bioactive and functional properties (Manso and López-Fandiño, 2004). The nonglycosylated CMP form is referred to as aglyco-CMP (aCMP) and the glycosylated form as glyco-CMP (gCMP). The abundant aCMP forms of variants $\mathrm{A}, \mathrm{B}$, and $\mathrm{E}$ containing 1 phosphorylation (VarA:1P, VarB:1P, and VarE:1P) constitute the reference masses, with molecular weights $(\mathrm{Mw})$ of $6,787.62,6,755.63$, and 6,757.63 Da, respectively.

Functional properties such as surface tension, foam rigidity, and stability are reduced in food matrices that contain gCMP compared with matrices that contain aCMP (Kreuß et al., 2009a, b). Additionally, $\mathrm{N}$-acetylneuraminic acid (NeuAc)-containing gCMP has also been associated with the formation of unwanted storage-induced aggregates in acidic whey beverages (Villumsen et al., 2015; Le et al., 2016). It is characterized by a high degree of heterogeneity, because it originates from different genetic variants of $\kappa$-casein with a variety of post-translational modifications. At least 11 genetic variants of $\kappa$-casein have been identified in cattle, referred to as A, B, C, E, F $\mathrm{F}^{1}, \mathrm{~F}^{2}, \mathrm{G}^{1}, \mathrm{G}^{2}, \mathrm{H}$, I, and J (Farrell et al., 2004). The 2 dominating genetic variants in bovine milk are A and B. The B variant differs from the A variant by 2 substitutions, at amino acid residues 136 $($ Thr $\rightarrow$ Ile) and 148 (Asp $\rightarrow$ Ala; Farrell et al., 2004). Signal peptide is not included in the reported positions. Further, variant E, which is also widely reported in different breeds, differs from variant A by a substitution of amino acid residue 155 (Ser $\rightarrow$ Gly). Knowledge of the heterogeneity in post-translational modifications is crucial to fully understanding the functional and physiological properties of CMP. Previous studies have 
reported that $\kappa$-casein isoforms contain 1 to 6 O-linked glycosylations and 1 to 3 phosphorylations (Holland et al., 2006). The possible O-linked glycosylation sites on א-casein are at positions $\mathrm{Thr}^{121}, \mathrm{Thr}^{131}, \mathrm{Thr}^{133}, \mathrm{Thr}^{136}$ (only in CMP variants A and $\mathrm{E}$ ), $\mathrm{Thr}^{142}$, $\mathrm{Thr}^{145}$, and $\mathrm{Th}^{165}$ (Saito and Itoh, 1992; Holland et al., 2006). Glycan addition to the polypeptide seems to occur in a certain sequence, with the primary glycosylation site being $\mathrm{Thr}^{131}$, the second at $\mathrm{Thr}^{142}$, and the third at $\mathrm{Thr}^{133}$ (Holland et al., 2005). However, a more recent study by Nwosu et al. (2010) showed equal degrees of glycosylation at $\mathrm{Thr}^{131}$ and $\mathrm{Thr}^{133}$, and all glycosylation forms associated with these positions. Phosphorylation sites are at positions $\mathrm{Ser}^{127}$, Thr ${ }^{145}$, and $\mathrm{Ser}^{149}$ (Holland et al., 2004).

The reported content of glycosylated versus nonglycosylated $\kappa$-casein in bovine milk varies across studies. Up to 50 to $60 \%$ has been estimated to be glycosylated (Vreeman et al., 1986; Farrell et al., 2004), although more recent studies have revealed lower values, at 17 to 37\% (Jensen et al., 2012a; Bijl et al., 2014; Poulsen et al., 2016). The O-linked glycosylations on CMP in mature bovine milk consist of the neutral monosaccharides $N$-acetyl-galactosamine (GalNAc) and galactose (Gal), and the acidic monosaccharide NeuAc, commonly referred to as sialic acid (Manso and López-Fandiño, 2004). The CMP isoforms from bovine colostrum are more heterogeneous than isoforms from mature bovine milk, also containing fucose and $\mathrm{N}$-acetyl-glucosamine (GlcNAc; O'Riordan et al., 2014). The following 5 predominating $\kappa$-casein glycosylation forms are denoted a to e, as previously shown by Saito and Itoh (1992): a $=$ GalNAc $(\mathrm{Mw}=203.19 \mathrm{Da}) ; \mathrm{b}=\mathrm{Gal} \beta(1-3)$ GalNAc $(\mathrm{Mw}=365.34 \mathrm{Da}) ; \mathrm{c}=\operatorname{NeuAc} \alpha(2-3) \mathrm{Gal} \beta(1-3) \mathrm{Gal}-$ NAc $(\mathrm{Mw}=656.59 \mathrm{Da}) ; \mathrm{d}=\operatorname{Gal} \beta(1-3)[\operatorname{NeuAc} \alpha(2-6)]$ GalNAc $(\mathrm{Mw}=656.59 \mathrm{Da})$; and $\mathrm{e}=\operatorname{NeuAc\alpha }(2-3)$ Gal $\beta(1-3)[$ NeuAc $\alpha(2-6)]$ GalNAc $(\mathrm{Mw}=947.85$ Da $)$. More recently, an additional simple glycosylation form has been observed constituting GalNAc and NeuAc $(\mathrm{Mw}=494.45 \mathrm{Da}$; Nwosu et al., 2010). The glycan structures a to e have been reported to be present in amounts of approximately 1, 6, 18, 19, and 56\%, respectively (Saito and Itoh, 1992). The high degree of sialylation may affect functional properties, mainly due to electrostatic interactions (Villumsen et al., 2015), so an enzymatic desialidation of gCMP is expected to change its contribution to food structure. The objective of the present work was to characterize gCMP isoform heterogeneity before and after sialidase treatment of commercial bovine gCMP, using 2-dimensional gel electrophoresis (2-DGE) and liquid chromatography/mass spectroscopy (LC-ESI/MS), based on combinations of retention times (RT), masses, and isoelectric point (pI). Findings from the present work will provide ana- lytical tools to enable simple validation of desialidation for future functionality studies.

The desialidation procedure was conducted in duplicates of CMP solutions based on the commercial Lacprodan cGMP-20 isolated from sweet cheese whey (Arla Foods Ingredients, Denmark) and obtained as spray-dried powder. The CMP powder was reconstituted at $7 \%$ (wt/vol) prepared in Milli-Q water with $0.02 \%$ sodium azide (wt/vol). A commercial sialidase (EC 3.2.1.18) enzyme preparation, from Clostridium perfringens (Sigma Aldrich, St. Louis, MO), was used for the desialidation, with an activity of $100 \mathrm{U} / \mathrm{mg}$ of enzyme. The samples were adjusted to $\mathrm{pH} 5.8$ to reach the optimum conditions for the enzyme, according to the manufacturer. Then, the sialidase was added to the solution in an enzyme:substrate ratio of 1:57,000 (wt/ wt), corresponding to $1 \mathrm{U} / 570 \mathrm{mg}$ of gCMP isolate. The sialidase-treated samples were incubated at $37^{\circ} \mathrm{C}$ for $24 \mathrm{~h}$. The CMP solutions before and after desialidation were analyzed in triplicate using 2-DGE under reducing conditions. A $25-\mu \mathrm{L}$ aliquot of each CMP isolate solution was diluted in lysis buffer $(\mathrm{pH} 7.5)$, containing $7 M$ urea, $2 M$ thiourea, 4\% 3-[(3-cholamidopropyl) dimethylammonio]-1-propanesulfonate, $1 \%$ 1,4-dithioerythritol (DTE), and $40 \mathrm{~m} M$ Tris- $\mathrm{HCl}$, to a final protein concentration of $300 \mu \mathrm{g}$ in $200 \mathrm{~mL}$. Because the present study was focusing on the proteomic analysis of CMP, which has a pI of 3.15 to 4.15 (Kreuß et al., 2009b), the samples were focused using immobilized $\mathrm{pH}$ gradient strips (pH 3-6, $11 \mathrm{~cm}$; BioRad, Hercules, CA) and electrophoresed using SDS polyacrylamide gels (Criterion, TGX Stain-free Precast gels; BioRad), according to Jensen et al. (2012b). Proteins were stained with colloidal Coomassie Blue G-250, and glycoproteins were then identified by periodic acid-Schiff (PAS) staining. Gel images were obtained using an UVP Multispectral Imaging System (BioSpectrum, Cambridge, UK).

We conducted further profiling and molecular identification of native and desialylated CMP isoforms using LC-ESI/MS, essentially as described by Le et al. (2016). All samples were diluted to $3.5 \%$ CMP to aliquots of $250 \mu \mathrm{L}$. Samples were then diluted in $6 \mathrm{M}$ guanidine hydrochloride and reduced by $15 \mathrm{mM}$ DTE at $37^{\circ} \mathrm{C}$ for $60 \mathrm{~min}$. The analysis was performed on an Agilent LC 1100 series instrument (Agilent Technologies, Palo Alto, CA), where aliquots of $20 \mu \mathrm{L}$ (denatured and reduced) were injected onto the LC-ESI/MS system. The CMP were separated on a Jupiter C4 column ( $5 \mu \mathrm{m}$, $250 \mathrm{~mm} \times 2 \mathrm{~mm}$; Phenomenex, Torrance, CA) operated at $40^{\circ} \mathrm{C}$. Solvent A was Milli-Q water with $0.05 \%$ (vol/vol) trifluoroacetic acid and solvent B was acetonitrile with $0.05 \%$ ( vol $/ \mathrm{vol}$ ) trifluoroacetic acid. The gradient was started at $20 \% \mathrm{~B}$ for 5 min and increased 
to $50 \%$ B for $80 \mathrm{~min}$ with a flow rate of $0.3 \mathrm{~mL} / \mathrm{min}$ to achieve optimal resolution of CMP forms. Peptides were detected by UV absorbance at $214 \mathrm{~nm}$ coupled with the single-Q ESI/MS system (Agilent Technologies). The MS fragmentor was set at $150 \mathrm{~V}$, and the MS was run in positive mode from 400 to $1,800 \mathrm{~m} / z$. We conducted the qualitative and quantitative analyses using ChemStation software (rev. B.04.01 SP1; Agilent Technologies). Mass spectra were deconvoluted, and weighted average masses were obtained from the MS chromatograms. The CMP isoforms were identified by comparing their deconvoluted experimental masses to theoretical masses using the in-house library for CMP isoforms, as reported by Jensen et al. (2015). Relative quantification of the polypeptides was obtained as integrated peak area relative to the total integrated area (RT 17-43 min) in the UV chromatograms.

The electrophoretic characterization of CMP by 2-DGE is shown in Figure 1. The figure reveals CMP solutions before (A and $\mathrm{C}$ ) and after $(\mathrm{B}$ and $\mathrm{D})$ desialidation, and stained with Coomassie Blue (A and B) or PAS (C and D). Only the region from $\mathrm{pI} 3.0$ to 4.5 is shown. From the 2-DGE gels, it can be seen that the native CMP isolate contains a variety of isoforms, with the major spots labeled 1 to 8 . We observed minor spots at pI 4 and onwards. All replicated gels revealed similar distribution of spots. The molecular mass of monomeric CMP is approximately $6.7 \mathrm{kDa}$, although the CMP appeared as trimers in the gels, with estimated masses of 19 to $25 \mathrm{kDa}$. Similar findings have been observed in previous studies (Silva-Hernandez et al., 2002; Nakano et al., 2007; Farías et al., 2010), although the mechanism behind the appearance of these aggregates is not known. Based on the pI, masses, and positions of CMP isoforms from previous studies, the present work provides an estimated determination of the spots. Spots 1 and 2 contain molecules in the mass range of 23 to 25 $\mathrm{kDa}$ and $\mathrm{pI} 3.0$ to 3.1 ; spots 3 to 6 in the mass range of 19 to $21 \mathrm{kDa}$ and pI 3.6 to 4.0 ; and spots 7 and 8 in the mass range of 23 to $25 \mathrm{kDa}$ and $\mathrm{pI} 3.7$ to 3.9. Spots 5 and 6 were expected to consist mainly of VarA/E:1P and VarB:1P with pI 3.8 and 4.0, respectively, because variants $A$ and $E$ are more acidic than variant $B$ due to the $\beta$-carboxylic acid group on $\mathrm{Asp}^{148}$, compared with Ala ${ }^{148}$ (Holland et al., 2006). Spots 3 and 4 were expected to be VarA/E:2P and VarB:2P, with pI of 3.6 and 3.7, respectively.

On the PAS-stained gels, only spots 1 and 2 were observed before sialidase treatment (Figure 1C) and only spots 7 and 8 after treatment (Figure 1D). This shift in $\mathrm{pI}$ reflects the cleavage of negatively charged NeuAc residues. The pI of sialylated gCMP has previously been reported to be 3.1 as a result of the high degree of sialylation and the low $\mathrm{p} K_{\mathrm{a}}$ of NeuAc $(2.2$; Farías et al., 2010). The aCMP is less acidic, and has a pI of approximately 4.0 to 4.15 (Kreuß et al., 2009b; Svanborg et al., 2016). Previous studies have shown that pI decreases 0.07 to 0.31 for each NeuAc residue cleaved off (Barrabés et al., 2010). The observed shift in $\mathrm{pI}$ on the 2-DGE gels was approximately 0.9 , which may indicate that 3 to $13 \mathrm{NeuAc}$ residues are cleaved off on average, a realistic range for glycosylated trimers. Because staining of the glycosylated polypeptides was generally weak, spots other than those emphasized may also have contained glycosylations.

The molecular characterization by LC-ESI/MS revealed further identification of the CMP isoforms before and after desialidation and confirmed the findings from the 2-DGE gels. The analysis identified at least 19 distinct CMP isoforms, of which 13 were glycosylated and 6 were nonglycosylated (Table 1 ). Figure $2 \mathrm{~A}$ shows a chromatographic overlay of the CMP profiles in native and desialidated isolates. Two major peaks
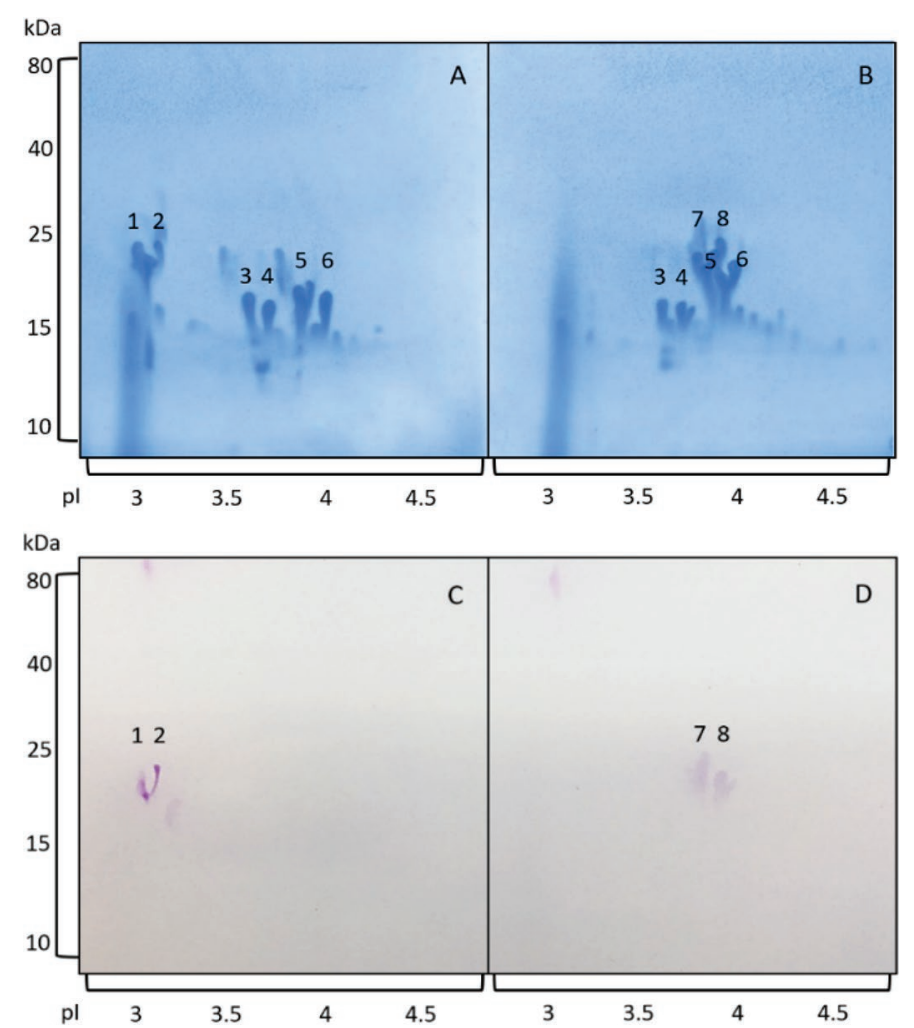

Figure 1. Reducing 2-dimensional gel electrophoresis of caseinomacropeptide (CMP) isolate solutions. (A) and (B) Polypeptides stained with Coomassie Blue. (C) and (D) Glycopeptides stained with periodic acid-Schiff stain. (A) and (C) Untreated CMP solution 7\% protein (wt/vol). (B) and (D) Sialidase-treated CMP solution in an enzyme:substrate ratio of 1:57,000 (wt/wt). Major gel spots are labeled 1 to $8 . \mathrm{pI}=$ isoelectric point. 
were identified at RT 32.2 and $42.4 \mathrm{~min}$. The peak eluting at 32.2 min was the aCMP isoforms VarA:1P and VarE:1P, and the peak eluting at 42.4 min was the aCMP isoform VarB:1P. We observed no changes in elution time or amount for these peaks after the enzymatic treatment. The content of aCMP in the present study was approximately $63 \%$, based on the area of nonglycosylated peaks relative to the total area under the curve. The chromatographic areas constituting peaks eluting between 15 and $31 \mathrm{~min}$ and 34 and 40 min were mainly found to contain gCMP isoforms. One exception was the aCMP isoform of variant $\mathrm{A}$ with 2 phosphorylations (VarA:2P): this isoform was observed in the area marked for gCMP (27.5 min; Figure 2A), which indicated different phosphorylation sites for the diphosphorylated forms. Figures $2 \mathrm{~B}$ and $2 \mathrm{C}$ reveal the identified CMP isoforms before and after sialidase treatment. The corresponding deconvoluted masses obtained by MS are shown in Table 1. The isotopic pattern of the CMP isoforms was in accordance with the theoretical distribution, as well as with previous studies (Le et al., 2013; Jensen et al., 2015).

In the present study, approximately $37 \%$ of the native CMP were glycosylated, in agreement with previous findings (Jensen et al., 2012b; Poulsen et al., 2016). A high content of glycosylated $\kappa$-casein relative to total protein has been associated with a small average casein micelle size (Bijl et al., 2014), and seemed to increase over lactation in milk from Danish Holstein-Friesian cows (Poulsen et al., 2016). We identified all 3 genetic variants of aCMP as both mono- and diphosphorylated isoforms. Up to 3 phosphorylations have been detected previously in aCMP fractions of $\kappa$-casein (Vreeman et al., 1986; Holland et al., 2006). We did not identify any triphosphorylated forms; this may have been due to the highly negative charges of these residues, which may be difficult to detect in the positive ion mode. Based on integrated peak areas, the relative content of monophosphorylated aCMP:1P in the untreated samples was $53.8 \%$ and the relative content of diphosphorylated

Table 1. Identification of caseinomacropeptide (CMP) isoforms present in the liquid chromatography-electrospray ionization-mass spectroscopy (LC-ESI/MS) chromatograms, detected in native and sialidase-treated CMP isolate

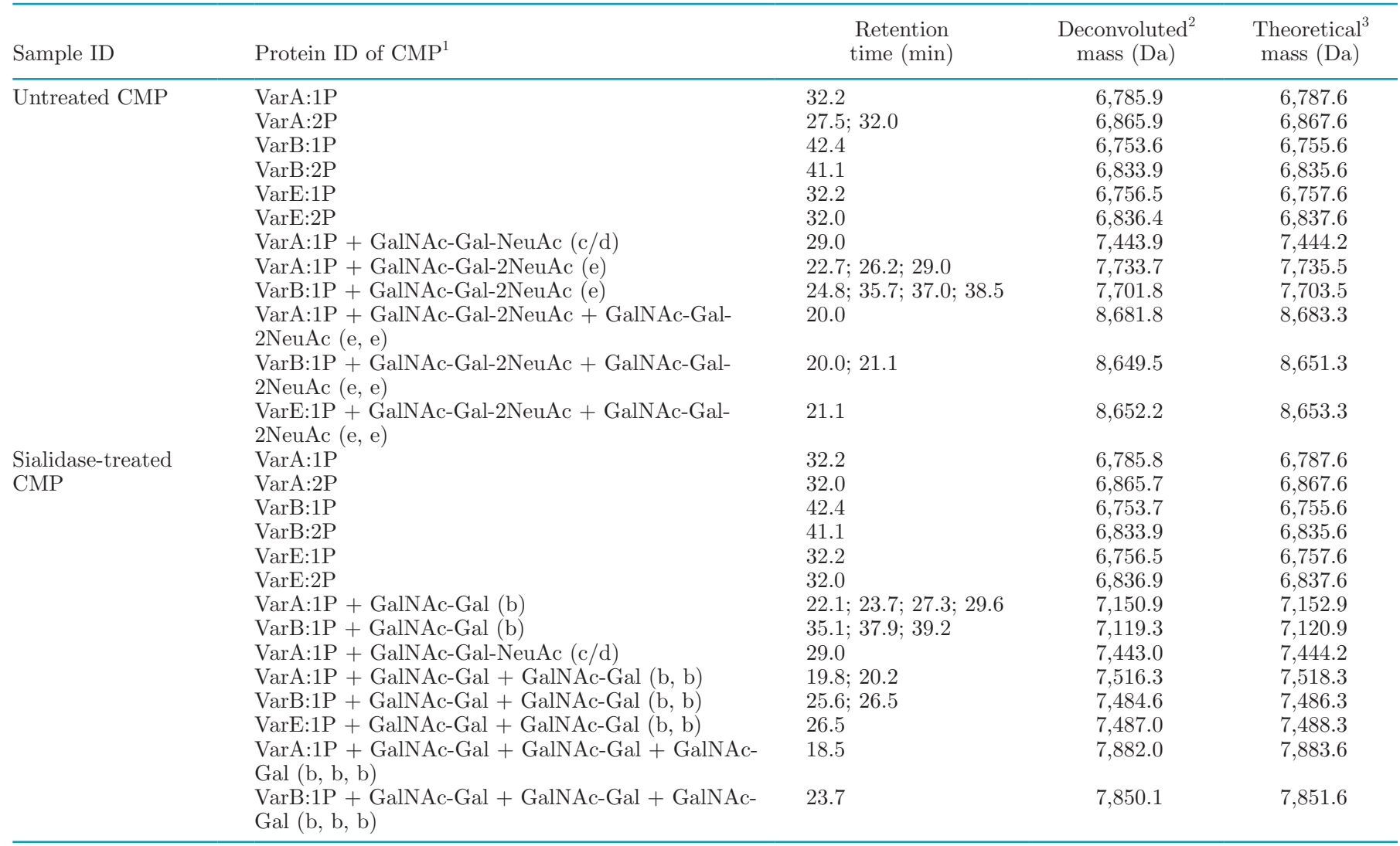

\footnotetext{
${ }^{1}$ Lowercase letters refer to the 5 predominating glycosylation forms. It is not possible to differentiate between the $\mathrm{c}$ and $\mathrm{d}$ forms based on the masses alone, so c/d denotes either of these forms.

${ }^{2}$ Masses detected by LC-ESI/MS.

${ }^{3}$ Based on in-house library as reported by Jensen et al. (2015).
} 

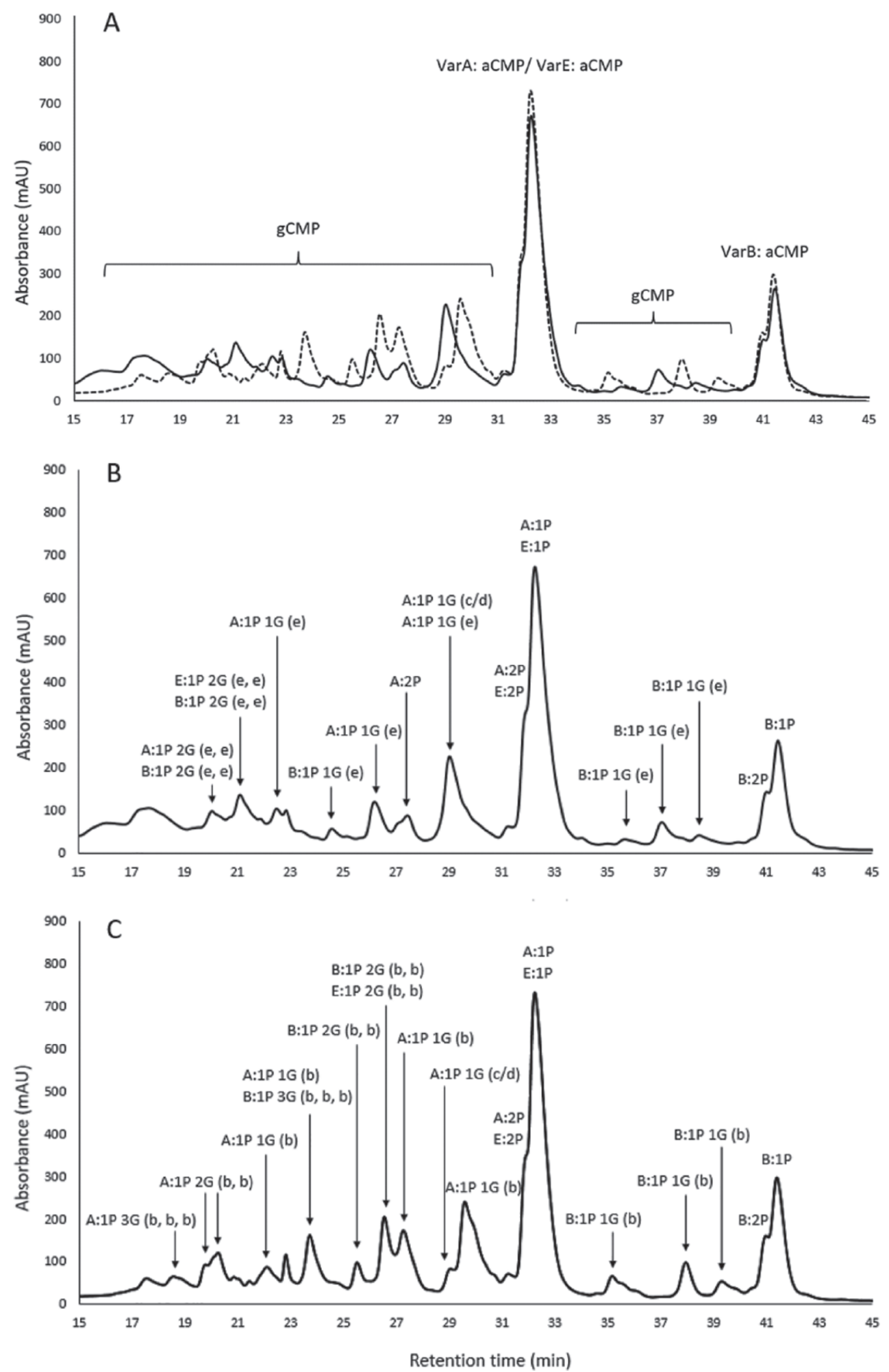

Figure 2. Effect of sialidase treatment on glycosylated caseinomacropeptide (gCMP) isoforms shown by liquid chromatography/mass spectroscopy (LC-MS). (A) Overlay of liquid chromatograms illustrating CMP solutions before (solid line) and after (dotted line) sialidase treatment. The CMP isoforms are labeled as gCMP, VarA:aCMP (variant A of nonglycosylated CMP), VarE:aCMP (variant E of nonglycosylated CMP), and VarB:aCMP (variant B of nonglycosylated CMP). Annotated liquid chromatograms of CMP isoforms in (B) native CMP isolate and (C) sialidase-treated CMP isolate. Identified CMP isoforms are denoted by genetic variant (A, B, E), number of phosphorylations (P), number of glycosylations $(\mathrm{G})$, and glycosylation forms (a, b, c, d, e). Peaks are identified by mass spectrometry, and deconvoluted masses are shown in Table 1. $\mathrm{mAU}=$ milli-arbitrary units. 
aCMP:2P was $9 \%$. This was in line with Jensen et al. (2012a), who reported that 45 to $50 \%$ of nonglycosylated $\kappa$-caseins were monophosphorylated, and 12 to $15 \%$ were diphosphorylated in Danish Holstein-Friesian and Danish Jersey milk. We identified no diphosphorylated gCMP isoforms in the present study. The absence of diphosphorylated gCMP was in agreement with Jensen et al. (2015) and Le et al. (2016), whereas Guerrero et al. (2015) detected diphosphorylated gCMP isoforms containing the minor glycans $\mathrm{b}$ and $\mathrm{c} / \mathrm{d}$.

In the present work, all gCMP isoforms detected in the native CMP isolate seemed to contain NeuAc (Table 1), consistent with the proteomic profile observed in the 2-DGE gels. Only 1 isoform was monosialylated, containing a single NeuAc residue. This compound was identified as VarA:1P (c/d) (29 min; Figure 2B), with a molecular mass of 7,443.9 Da. Based on previous knowledge of the distribution of the 5 predominating glycosylations attached to CMP, we expected that the major part of gCMP would be disialylated. In comparison, Guerrero et al. (2015) identified VarA and VarB containing $\mathrm{c} / \mathrm{d}$ glycosylations in a gCMP isolate, both as mono- and diphosphorylated forms. As well, Jensen et al. (2015) identified multiglycosylated gCMP isoforms, containing $\mathrm{c} / \mathrm{d}$ glycosylations combined with e glycosylations. These forms may also be present in the CMP isolate eluting at low RT. It has been suggested that the major glycan on $\kappa$-casein is the tetrasaccharide containing 2 NeuAc residues (e glycosylation), and that detection of the minor glycans might be a result of incomplete synthesis of the tetrasaccharide, or degradation during processing or analysis preparation (Holland et al., 2004). This points to a lower natural content of monosialylated glycans on $\kappa$-casein than often reported, and fits well with the high level of detected disialylated glycans in the present work. Several CMP isoforms with identical masses eluted at distinct RT, indicating different positional isomers. An example is $\operatorname{VarA}: 1 \mathrm{P}(\mathrm{b})$, which eluted at 4 different RT, corresponding to 4 different isomers. The sites of glycosylation reveal variations in hydrophobicity and thereby RT. The same was observed for VarB:1P (e). Further, VarA:1P (e) and VarB:1P (b) were identified each in 3 peaks in the chromatograms. Because the exact site of glycosylation affects the hydrophobicity of the molecules, these would also be expected to influence cleavage-specific properties (e.g., of chymosin), or functional properties of gCMP in food products, even with the same glycans attached.

In total, we identified 2 glycosylated forms of the genetic variant $\mathrm{E}$ in the solutions before and after sialidase treatment. The genetic variant $\mathrm{E}$ was VarE:1P (e, e) in the native CMP isolate (21.1 min; Figure 2B), and VarE:1P $(\mathrm{b}, \mathrm{b})$ in the sialidase-treated CMP solution (26.5 min; Figure 2C). The isoform VarE:1P (e, e) was also identified by Jensen et al. (2015) in milk from Danish Holstein-Friesian cows. Only few other studies have reported on different isoforms of the CMP VarE. Because VarE:1P (b, b) was only detected in the sialidase-treated solutions, these glyco-peptides seem to be the same compounds observed before and after cleavage of a total of $4 \mathrm{NeuAc}$ residues, resulting in formation of 2 disaccharides from 2 tetrasaccharides. Thus, the dominating gCMP isoform of genetic VarE seems to contain 4 NeuAc residues, whereas the dominating gCMP isoforms of VarA and VarB seem to contain 2 NeuAc. These findings may explain why VarE has previously been associated with poorly coagulating milk (Jensen et al., 2012a), because the highly negative charges will provide a repulsive effect and may decrease coagulation properties.

Up to 6 glycosylations can be present per gCMP molecule, and therefore up to 12 NeuAc residues can be attached per gCMP polypeptide (Holland et al., 2006). In the native CMP isolate, all gCMP seemed to contain NeuAc, so the content of sialylated gCMP relative to the total CMP content was $37 \%$, which after sialidase treatment was decreased to $1.5 \%$, based on the integrated area under the UV chromatogram. This indicates a $96 \%$ effectivity of desialidation. The presence of very few acidic gCMP isoforms after treatment might explain the observed smear in the $\mathrm{pI}$ range 3.0 to 3.1 (Figure 1B). Identification of the gCMP peaks revealed the following conversions after sialidase treatment. Peaks representing gCMP (VarA and B) with 1 c/d or e glycosylation were hydrolyzed to gCMP with $1 \mathrm{~b}$ glycosylation by cleavage of 1 or $2 \mathrm{NeuAc}$ residues $(\Delta \mathrm{m}=291 \mathrm{Da}$ per NeuAc residues), respectively (Table 1). Further, peaks corresponding to gCMP (VarA, $\mathrm{B}$, and $\mathrm{E}$ ) with 2 e glycosylations were hydrolyzed to gCMP with 2 b glycosylations by cleavage of 4 NeuAc residues. Two isoforms containing $3 \mathrm{~b}$ glycosylations were identified in the desialidated samples, although no sialylated counterparts were found in the native isolate. It is thus assumed that gCMP isoforms containing $3 \mathrm{e}$ glycosylations were present in the untreated samples. These isoforms would be expected to elute at very low RT, because the hydrophobicity is significantly decreased by the NeuAc residues, and may correspond to the 2 peaks observed at RT 15 to 19 min, which were not identified. As was probably also the case with the highly phosphorylated forms, these residues may be too negatively charged for the equipment to detect them. Previous studies have reported gCMP isoforms containing up to 3 e glycosylations (Holland et al., 2004; Jensen et al., 2015). The 3 positional isomers of VarB:1P (b) 
(RT 35-39 min, Figure 2C) after treatment are cleavage products of VarB:1P (e) (RT 35-39 min, Figure 2B). These peaks were higher and more separated after sialidase treatment, confirming the presence of several positional isomers in the CMP isolate. To our knowledge, no other studies have reported 3 VarB:1P (e) in this RT interval. These differences in findings across studies might be explained by differences in the product type analyzed. A commercial CMP isolate is expected to contain a higher degree of post-translational modifications and genetic variations than whey from bulk or tank milk, because it is a concentrated product originating from a variety of different herds and breeds, and possibly from different cheese production streams.

The present study shows that 2-DGE combined with LC-ESI/MS reveals a detailed elucidation of the hydrolysis pattern of gCMP by sialidase treatment. The proteomic analyses allowed us to characterize at least 19 distinct CMP isoforms; 3 genetic variants (A, B, and $\mathrm{E})$ were identified as both nonglycosylated and glycosylated isoforms, with up to 3 glycans per CMP and up to 4 glycosylation positions. We observed a more complex pattern of CMP isoforms after sialidase treatment than before treatment, both according to the UV chromatograms (Figure 2) and to the 2-DGE gels (Figure 1). The chromatogram revealed 6 different gCMP isoforms before treatment and 8 after treatment; further, 2-DGE revealed additional minor spots after enzymatic treatment in the pI range of 3.5 to 4.4. Although it was possible to follow the desialidation of specific gCMP isoforms, we could not detect the multiglycosylated gCMP isoforms containing combinations of the e and c/d glycosylations or up to 3 e glycosylations. However, the gCMP isoforms carrying 3 e glycosylations are expected to be present in both variants $\mathrm{A}$ and $\mathrm{B}$, because we detected isoforms containing $3 \mathrm{~b}$ glycosylations after sialidase treatment. We do not expect this lack of identification of minor forms of CMP to change our conclusions on the effect of desialidation. Functionality - and hence the stability of food products - is expected to be affected by certain residues, such as charged amino acids, phosphate groups, and NeuAc, because of their potential electrostatic interactions (Villumsen et al., 2015; Le et al., 2016). Because gCMP is a highly sialylated glycoprotein, NeuAc is expected to have a significant effect on the functionality properties of CMP-containing products (Thomä-Worringer et al., 2006). Desialidation may therefore change the functional properties of CMP. Results from the present work will be of great utility for future studies investigating its functionality and biochemical properties and provide relatively simple and effective analytical tools for the validation of desialidation of gCMP at the molecular level.

\section{ACKNOWLEDGMENTS}

The authors thank Arla Foods Ingredients for providing the CMP isolate and laboratory technicians Hanne Søndergård Møller and Gitte Hald Kristiansen at Aarhus University (Tjele, Denmark) for their highly valued contribution in the experimental phase of the work. The authors are also grateful for the financial support from Aarhus University Research Foundation (AUFF) and the Graduate School of Science and Technology (GSST) at Aarhus University.

\section{REFERENCES}

Barrabés, S., A. Sarrats, E. Fort, R. De Llorens, P. M. Rudd, and R. Peracaula. 2010. Effect of sialic acid content on glycoprotein pI analyzed by two-dimensional electrophoresis. Electrophoresis 31:2903-2912.

Bijl, E., R. de Vries, H. van Valenberg, T. Huppertz, and T. van Hooijdonk. 2014. Factors influencing casein micelle size in milk of individual cows: Genetic variants and glycosylation of $\kappa$-casein. Int. Dairy J. 34:135-141.

Farías, M. E., M. J. Martinez, and A. M. R. Pilosof. 2010. Casein glycomacropeptide $\mathrm{pH}$-dependent self-assembly and cold gelation. Int. Dairy J. 20:79-88.

Farrell, H. M., R. Jimenez-Flores, G. T. Bleck, E. M. Brown, J. E Butler, L. K. Creamer, C. L. Hicks, C. M. Hollar, K. F. Ng-KwaiHang, and H. E. Swaisgood. 2004. Nomenclature of the proteins of cows' milk — sixth revision. J. Dairy Sci. 87:1641-1674.

Guerrero, A., L. Lerno, D. Barile, and C. B. Lebrilla. 2015. Top-down analysis of highly post-translationally modified peptides by Fourier transform ion cyclotron resonance mass spectrometry. J. Am. Soc. Mass Spectrom. 26:453-459.

Holland, J. W., H. C. Deeth, and P. F. Alewood. 2004. Proteomic analysis of kappa-casein micro-heterogeneity. Proteomics 4:743-752.

Holland, J. W., H. C. Deeth, and P. F. Alewood. 2005. Analysis of O-glycosylation site occupancy in bovine kappa-casein glycoforms separated by two-dimensional gel electrophoresis. Proteomics 5:990-1002.

Holland, J. W., H. C. Deeth, and P. F. Alewood. 2006. Resolution and characterisation of multiple isoforms of bovine kappa-casein by 2-DE following a reversible cysteine-tagging enrichment strategy. Proteomics 6:3087-3095.

Jensen, H. B. J. W. Holland, N. A. Poulsen, and L. B. Larsen. 2012a. Milk protein genetic variants and isoforms identified in bovine milk representing extremes in coagulation properties. J. Dairy Sci. 95:2891-2903

Jensen, H. B., K. S. Pedersen, L. B. Johansen, N. A. Poulsen, M. Bakman, D. E. Chatterton, and L. B. Larsen. 2015. Genetic variation and posttranslational modification of bovine kappa-casein: Effects on caseino-macropeptide release during renneting. J. Dairy Sci. 98:747-758

Jensen, H. B., N. A. Poulsen, H. S. Moller, A. Stensballe, and L. B. Larsen. 2012b. Comparative proteomic analysis of casein and whey as prepared by chymosin-induced separation, isoelectric precipitation or ultracentrifugation. J. Dairy Res. 79:451-458.

Kreuß, M., I. Krause, and U. Kulozik. 2009a. Influence of glycosylation on foaming properties of bovine caseinomacropeptide. Int. Dairy J. 19:715-720.

Kreuß, M., T. Strixner, and U. Kulozik. 2009b. The effect of glycosylation on the interfacial properties of bovine caseinomacropeptide. Food Hydrocoll. 23:1818-1826.

Le, T. T., H. C. Deeth, B. Bhandari, P. F. Alewood, and J. W. Holland. 2013. Quantification of lactosylation of whey proteins in stored milk powder using multiple reaction monitoring. Food Chem. 141:1203-1210. 
Le, T. T., S. D. Nielsen, N. S. Villumsen, G. H. Kristiansen, L. R. Nielsen, S. B. Nielsen, M. Hammershøj, and L. B. Larsen. 2016. Using proteomics to characterise storage-induced aggregates in acidic whey protein isolate drinks. Int. Dairy J. 60:39-46.

Manso, M. A., and R. López-Fandiño. 2004. k-casein macropeptides from cheese whey: physicochemical, biological, nutritional, and technological features for possible uses. Food Rev. Int. 20:329-355.

Nakano, T., N. Ikawa, and L. Ozimek. 2007. Detection of sialylated phosphorylated $\kappa$-casein glycomacropeptide electrophoresed on polyacrylamide gels and cellulose acetate strips by the thiobarbituric acid and malachite green dye reactions. J. Agric. Food Chem. $55: 2714-2726$.

Nwosu, C. C., J. S. Strum, H. J. An, and C. B. Lebrilla. 2010. Enhanced detection and identification of glycopeptides in negative ion mode mass spectrometry. Anal. Chem. 82:9654-9662.

O'Riordan, N., M. Kane, L. Joshi, and R. M. Hickey. 2014. Structural and functional characteristics of bovine milk protein glycosylation. Glycobiology 24:220-236.

Poulsen, N. A., H. B. Jensen, and L. B. Larsen. 2016. Factors influencing degree of glycosylation and phosphorylation of caseins in individual cow milk samples. J. Dairy Sci. 99:3325-3333.

Saito, T., and T. Itoh. 1992. Variations and distribution of O-glycosidically linked sugar chains in bovine $\kappa$-casein. J. Dairy Sci. $75: 1768-1774$.
Silva-Hernandez, E. R., T. Nakano, and L. Ozimek. 2002. Isolation and analysis of $\kappa$-casein glycomacropeptide from goat sweet whey. J. Agric. Food Chem. 50:2034-2038.

Svanborg, S., A.-G. Johansen, R. K. Abrahamsen, R. B. Schüller, and S. B. Skeie. 2016. Caseinomacropeptide influences the functional properties of a whey protein concentrate. Int. Dairy J. 60:14-23.

Thomä-Worringer, C., J. Sørensen, and R. López-Fandiño. 2006. Health effects and technological features of caseinomacropeptide. Int. Dairy J. 16:1324-1333.

Villumsen, N. S., H. B. Jensen, T. T. Thu Le, H. S. Møller, R. T. Nordvang, L. R. Nielsen, S. B. Nielsen, J. Sørensen, M. Hammersh $\varnothing$ j, and L. B. Larsen. 2015. Self-assembly of caseinomacropeptide as a potential key mechanism in the formation of visible storage induced aggregates in acidic whey protein isolate dispersions. Int. Dairy J. 49:8-15.

Vreeman, H. J., S. Visser, C. J. Slangen, and J. A. M. Van Riel. 1986. Characterization of bovine kappa-casein fractions and the kinetics of chymosin-induced macropeptide release from carbohydrate-free and carbohydrate-containing fractions determined by high-performance gel-permeation chromatography. Biochem. J. 240:87-97. 